\title{
GRADING AND METASTABLE EFFECTS IN ADMITTANCE SPECTROSCOPY OF CIGS-BASED SOLAR CELLS
}

\author{
Decock K. ${ }^{1}$, Khelifi S. ${ }^{1}$, Pianezzi F. ${ }^{2}$, Tiwari A.N. ${ }^{2}$ and Burgelman M. ${ }^{1}$ \\ ${ }^{1}$ Dept. of Electronics and Information Systems (ELIS), University of Gent, St-Pietersnieuwstraat 41, B-9000 Gent, Belgium \\ ${ }^{2}$ Laboratory for Thin Films and Photovoltaics, EMPA, Überlandstrasse 129, 8600 Dübendorf, Switzerland \\ E-mail: Koen.Decock@elis.ugent.be
}

\begin{abstract}
$\mathrm{Cu}(\mathrm{In}, \mathrm{Ga}) \mathrm{Se}_{2}$-based (CIGS) solar cells have achieved efficiencies up to $20 \%$. Despite these excellent results, the understanding of the underlying mechanisms and the influence of defects on their performance is still incomplete. The determination of the energetic position of the defects and of their density of states is important. Admittance spectroscopy is an adequate technique for this.

By varying the external voltage during the measurement, the spatial position where the defect distribution is sensed can be varied. However, the application of external biases can lead to metastable effects in the absorber and therefore to defect relaxation and changes in the doping distribution. Hence, it is important to separate between the effects caused by metastable changes and the change in sensing position of the admittance spectroscopy measurement. This can be achieved by varying the applied voltage during the creation of the metastable state and the measurement itself independently or simultaneously.
\end{abstract}

Admittance spectroscopy under different bias voltage conditions performed on a flexible CIGS-based solar cell are presented and assessed.

\section{INTRODUCTION}

$\mathrm{Cu}(\mathrm{In}, \mathrm{Ga}) \mathrm{Se}_{2}$-based (CIGS) solar cells have achieved efficiencies up to $20 \%$. Despite these excellent results, the understanding of the underlying mechanisms and the influence of defects on their performance is still incomplete. The determination of the energetic position of the defects and of their density of states is important.

Admittance spectroscopy is one of the techniques which allows to gain insight in the energetic position and the density of states of the defects present in a solar cell device. The technique is based on the analysis of capacitance measurements as a function of frequency and temperature and yields information about the defect density at the spatial position where the defect level intersects the Fermi level. Often defect distributions rather than discrete defect levels are present. A convenient way to interpret admittance measurements performed under zero bias conditions on samples exhibiting defect distributions has been introduced by Walter et al. [1]. This technique is based on calculating the derivative of the capacitance with respect to the measurement frequency and results in a graph of the defect density as a function of the defect level energy. This technique has recently been extended to non-zero bias conditions [2] which enhances its applicability, helps to improve and assess the accuracy of the results [3] and enables to extract additional information from the measurements.

CIGS-based solar cell absorbers often exhibit large nonuniformities. Some of these are even intentionally introduced, e.g. band gap grading by varying the In/Ga ratio [4]. Hence it is interesting to measure the defect density at various positions in the absorber. This is possible by changing the position of the Fermi level by applying an external bias voltage.

Native defects in $\mathrm{Cu}(\mathrm{In}, \mathrm{Ga}) \mathrm{Se}_{2}$ are a source of metastable effects [5] which are induced by illumination or by the application of an external bias voltage. Various admittance spectra measured with different external biases applied are hence mutually different not only because the defect density is measured at a different spatial position but also because defect relaxation can have changed the defect distribution. It is important to be able to separate the effects originating from grading and from metastable effects.

\section{THEORY}

The technique developed by Walter et al. [1] extracts the defect density distribution as a function of the defect energy level from the derivative of the capacitance to the (logarithm of the) measurement frequency.

A certain defect level can only be detected by admittance spectroscopy when the Fermi level intersects the defect level. The position in the sample where this intersection occurs is dependent on the applied voltage and the band bending. The energy $E_{\omega}$ which is measured in admittance spectroscopy corresponds to the energetic distance of the defect level to the closest band gap edge (unless the capture cross sections are very asymmetric).

In order to calculate the defect density from the derivative of the capacitance an assumption on the band bending is necessary. For CIGS-based solar cells it is adequate to assume parabolic band bending [2, 3]. In this case, the position of the defect energy level with respect to the valence band is given by Eq.(1) and shown in Fig. 1. The 


\section{$37^{\text {th }}$ IEEE PVSC - Seattle 2011 - DOI: 10.1109/pvsc.2011.6186187}

defect density can be calculated from the derivative of the capacitance according to Eq.(2).

$$
\begin{gathered}
E_{\omega}=E_{F n \infty}-q V-q\left(V_{b i}-V\right) \frac{x}{w}\left(2-\frac{x}{w}\right) \\
N_{t}\left(E_{\omega}\right)=-\frac{2\left(V_{b i}-V\right)^{3 / 2}}{w \sqrt{q} \sqrt{q V_{b i}-E_{F n \infty}+E_{\omega}}} \frac{d C}{d \omega} \frac{\omega}{k T}
\end{gathered}
$$

Assuming an $\mathrm{n}^{+} \mathrm{p}$ hetero-junction, $E_{F n \infty}$ represents the Fermi level position in the bulk of the n-side of the junction. $V$ represents the applied bias voltage, $V_{b i}$ the built-in voltage, $q$ the elementary charge, $w$ the depletion width and $x$ the position in the sample. $N_{t}$ represents the defect density and $C$ represents the capacitance measured with a test signal with an angular frequency $\omega$.

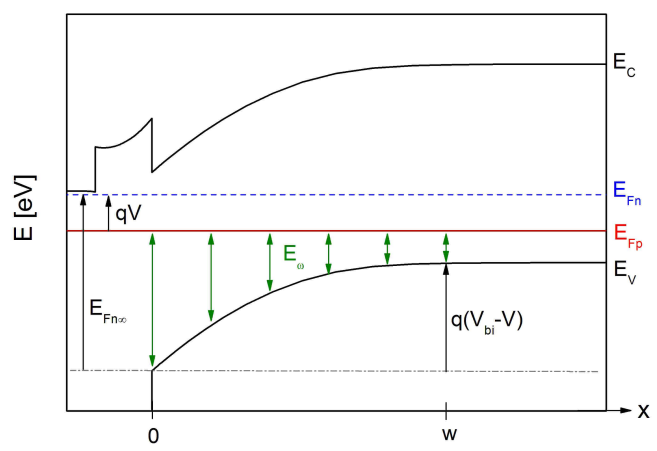

Figure 1: Schematic band diagram of a CIGS solar cell with an applied voltage $V$. The defect energy $E_{\omega}$, Eq. (1), sensed by admittance spectroscopy at several positions is indicated with green arrows.

Eq. (1) expresses all combinations of defect energy level values and the positions at which these defects are detected when the defect level is located close to the valence band. When the defect is located close to the conduction band it is more likely that the defect state gets charged/discharged from/to the conduction band. The defect energy $E_{\omega}$ which will be sensed will then no longer correspond to the distance between the valence band and the hole Fermi level, but rather to the distance between the conduction band and the electron Fermi level and is given by Eq.(3). Eq.(2) remains valid.

$$
E_{\omega}=E_{g}-E_{F n \infty}+q\left(V_{b i}-V\right) \frac{x}{w}\left(2-\frac{x}{w}\right)
$$

An overview of the combinations of $E_{\omega}$ and $x$ which can be measured at a certain bias voltage is given in Fig. 2 .

If the defect level is located close to the valence band, the position at which it is detected approaches the bufferabsorber interface when the applied bias voltage is increased. If the defect level is located close to the conduction band, this position moves towards the absorber bulk.

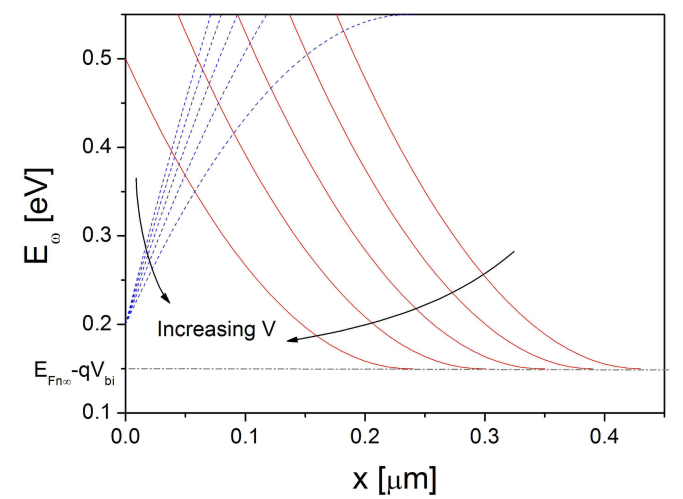

Figure 2: Overview of the position $x$ where a defect level $E_{\omega}$ is detected, according to Eq.(1) (close to $E_{V}$, red solid lines) and Eq.(3) (close to $E_{C}$, blue dashed lines). Calculation made assuming $E_{g}=1.1 \mathrm{eV}$; $E_{F n \infty}=0.9 \mathrm{eV} ; V_{b i}=0.75 \mathrm{eV} ; w=0.4 \mu \mathrm{m} \times\left(V_{b i}-V\right)^{1 / 2} . V=-0.4 \mathrm{~V}$ $\rightarrow 0.4 \mathrm{~V}$.

\section{METASTABLE DEFECT BEHAVIOUR}

The metastable changes in capacitance measurements can be assigned to thermally activated changes in the configuration of defects, where each configuration exhibits its own specific defect energy levels. The most important origins of metastabilities in the CIGS material system are the $\left(\mathrm{V}_{\mathrm{Se}}-\mathrm{V}_{\mathrm{Cu}}\right)$ complex and the $\left(\mathrm{In}_{\mathrm{Cu}}-n \mathrm{~V}_{\mathrm{Cu}}\right)$ complexes [6, 7]. As these changes in the configuration of the defect are thermally activated, they can be changed by varying the illumination conditions and applied voltage during an initial treatment at elevated temperatures. The distribution over the different configurations can then be assumed to remain constant when cooling down the sample. This distribution is governed by the capture cross-sections and activation energies of the processes involved in the transitions between the two configurations. From detailed balance calculations, a transition energy can be calculated which has similar properties as the defect level energy of a regular defect level. If the Fermi level energy during the initial treatment is above this transition energy, most of the defects will be in the acceptor configuration. If the Fermi level energy is below, most of the defects will be in the donor configuration, the fraction of defects still in the acceptor concentration decreases exponentially with the distance between the Fermi-level energy and the transition energy. As a result, the transition region from acceptor to donor configuration is very narrow.

At a certain position $x$ in the sample the fraction of metastable defects in the acceptor configuration depends on the Fermi level position during the initial condition. The defect energy which can be detected at this position depends on the Fermi level position during the capacitance measurement. There are three main approaches which each give different information about the sample. 


\section{$37^{\text {th }}$ IEEE PVSC - Seattle 2011 - DOI: 10.1109/pvsc.2011.6186187}

The most straightforward approach is to keep the sample in the same metastable state (same initial condition) and perform $C$-f-measurements with different bias voltages applied. In this case one gets the defect distribution at several positions for one given distribution of the metastable defects. The fraction of defects in the acceptor /donor configuration at a given position remains constant. Differences between the different measurements in this case are due to spatial variations in the defect density and in the spatial distribution of the metastable defects over acceptor and donor configuration. When the activation energy of the configurational change is low however the metastable state can have changed even at low temperatures.

A second approach is to vary the metastable state of the defects, whilst performing the measurements at one and the same bias voltage. Ideally this should give an insight in how the distribution of the defect of over acceptor and donor configuration varies at a certain position. However, as a difference in the metastable state of the defects changes the band bending in a sample, the assumption that all measurements yield information about the same positions is not always valid and difficult to verify.

A third approach is to vary the voltage during the initial condition and the measurement simultaneously. A main benefit of this approach is the certainty that the metastable state is conserved even if some activation energies are low. Moreover it allows to access the total defect density of the metastable defect, independent of the local distribution over the acceptor donor configuration. Consider e.g. a defect level belonging to the acceptor configuration of a metastable defect. This defect level can be detected using admittance spectroscopy at the position where its energy level intersects the Fermi level position. As the admittance is measured under the same conditions as the initial condition, this Fermi level position is determining the fraction of defects in the acceptor configuration as well. Hence for each measurement, the difference between the transition energy and the initial Fermi level energy is the same at the position where the defect density is sensed, and thus the fraction of defects in the acceptor state remains constant in all measurements. This way variations in the total metastable defect density can be measured independent of the metastable distribution between acceptor/donor configuration throughout the sample.

\section{EXPERIMENTAL}

Admittance measurements were performed on a flexible CIGS solar cell device $(n>15 \%)$ manufactured at EMPA. The sample was mounted in a $\mathrm{N}_{2}$-coolable cryostat varying the temperature between 100 and $360 \mathrm{~K}$ in steps of 20K. The frequency was varied from $100 \mathrm{~Hz}$ to $10 \mathrm{MHz}$. Data points which are hampered by parasitic network elements [8] or with an admittance phase angle smaller than $10^{\circ}$ have been discarded.
In a first measurement run (Fig. 3) the initial state of the sample was the same for all measurements (no illumination, bias voltage $V_{\text {init }}=0.0 \mathrm{~V}$ ) but the bias voltage for the individual measurements $\left(V_{\text {meas }}\right)$ was varied between $-2.0 \mathrm{~V}$ and $0.6 \mathrm{~V}$.

In a second run (Fig. 4) the initial state was obtained by keeping the sample at reverse bias $(-0.5 \mathrm{~V}$ and $-1.0 \mathrm{~V})$ without illumination. During measurement the applied bias voltage was kept constant to this value $\left(V_{\text {init }}=V_{\text {meas }}\right)$.

\section{RESULTS AND DISCUSSION}

As the initial state of all measurements in the first measurement is identical, it can be used to determine all parameters needed in Eq.(2). This results in $V_{b i}=1.06 \mathrm{eV}$ and $E_{F n^{\infty}}=0.9 \mathrm{eV}[3]$.

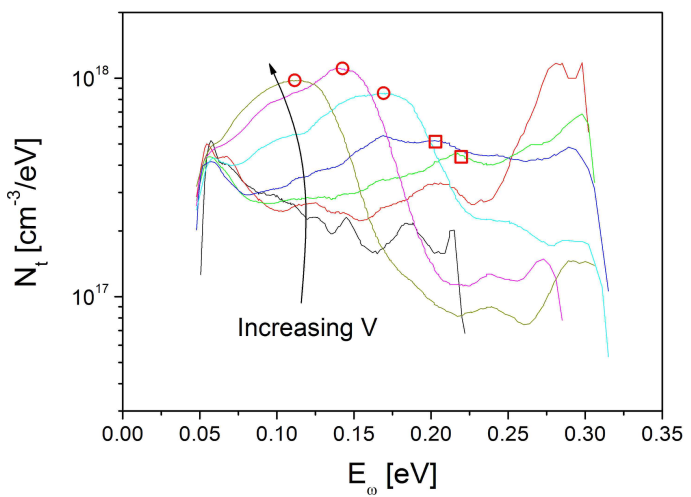

Figure 3: Overview of the apparent defect density measured with the same initial state at different bias voltages: $-2.0 \mathrm{~V} ;-1.5 \mathrm{~V} ;-1.0 \mathrm{~V} ;-0.5 \mathrm{~V} ; 0.0 \mathrm{~V} ; 0.3 \mathrm{~V}$ and $0.6 \mathrm{~V}$. [3]. Each curve is the result of several measurements at different temperatures, merged using a dedicated algorithm. The steep descent at $E_{\omega}<0.07 \mathrm{eV}$ is due to series resistance. The oscillations in the curve at high values of $E_{\omega}$ and at low voltage are due to the merging algorithm. Significant peaks are indicated with a circle, less significant peaks with a square.

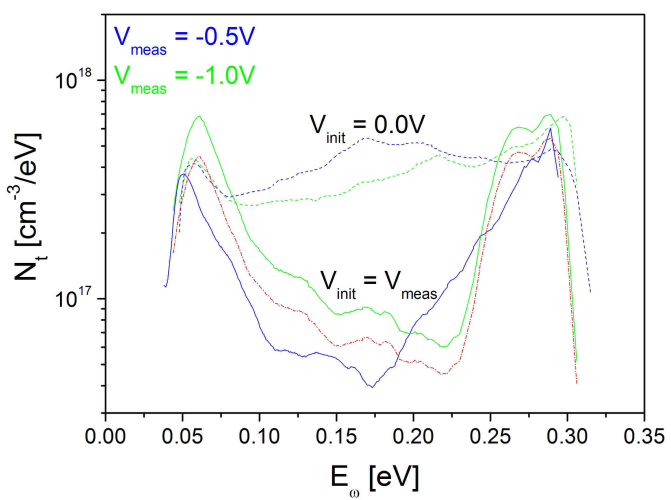




\section{$37^{\text {th }}$ IEEE PVSC - Seattle 2011 - DOI: 10.1109/pvsc.2011.6186187}

Figure 4: Overview of the apparent defect density measured with an applied bias voltage of $-0.5 \mathrm{~V}$ (blue) and $-1.0 \mathrm{~V}$ (green). The measurements with the same initial state, but different measurement bias voltage are drawn in dashed lines (see Fig. 3), the measurements where the bias during measurement is the same as in the initial state are drawn in solid lines. The red dash-dotted curve corresponds to the solid green curve with $E_{F n^{\infty}}=0.6 \mathrm{eV}$ instead of $0.9 \mathrm{eV}$. The peaks at $E_{\omega}<0.07 e V$ are due to series resistance. The steep descent at high values of $E_{\omega}$ is due to the merging algorithm.

In Fig. 3, a defect level is visible with $E_{\omega} \approx 0.1-0.2 \mathrm{eV}$ when $V_{\text {meas }} \geq-1.0 \mathrm{~V}$. $E_{\omega}$ decreases with increasing applied voltage. This energy region corresponds with capacitance measurement in the $160-240 \mathrm{~K}$ region.

The apparent defect density which is measured is strongly dependent on the initial and measurement bias voltage. The origin of the variation in the measurements can be explained assuming the defect to be the donor configuration of a DX-like metastable defect [7].

The donor configuration of this metastable defect exhibits a defect level close to the conduction band. The acceptor configuration is associated with a mid-gap level. The transition between the two configurations occurs at the point where the Fermi level during the initial state is approximately $0.9 \mathrm{eV}$ above the valence band, hence very close to the interface. The transition region between both configurations is very narrow.

As a result, when the $V_{\text {meas }}$ is large enough, the Fermi level will intersect the donor defect level and the defect can be detected. Under reverse measurement voltages, the intersection of the Fermi level with this energy level occurs in a region where the acceptor configuration is present instead of the donor configuration, and the defect level can not be detected. The change in defect level energy can be explained by the band gap grading, due to a large $\mathrm{Ga}$ grading in the sample near the interface [4].

The defect density for measurements with an initial state at $0.0 \mathrm{~V}$ is much larger than for the measurements with a reverse voltage during the initial state. When the activation energies related to the configurational change are large this behavior is not expected as the width of the region in the acceptor configuration is larger for more positive voltages during the initial state. For the DX-metastability however the activation energy for electron capture can be small (assuming a band gap of $1.04 \mathrm{eV}$, a pinning energy of $0.92 \mathrm{eV}$ and a activation energy of $0.32 \mathrm{eV}$ for electron emission [7], an activation energy of $0.08 \mathrm{eV}$ for electron capture is calculated from detailed balance considerations). Due to this low activation energy the metastable state can still change at lower temperatures. As the measurements in Fig. 3 were performed with increasing voltage at the different temperature steps, the metastable state of the defect can have been altered to a lower voltage initial state, leading to an increase of the measured defect density.

Comparing the measurements with $V_{\text {init }}=V_{\text {meas }}$, we expect no difference unless the total defect density (donor + acceptor) varies spatially. This behavior is not obvious in Fig. 4, at low energy values the apparent defect density differs for the different voltage conditions. As the metastable state is not equal however, $V_{b i}$ and $E_{F n^{\infty}}$ should not be equal as well, which influences the calculated apparent defect density. Changing the value of $E_{F n \text { no }}$ from $0.9 \mathrm{eV}$ to $0.6 \mathrm{eV}$ (red dash-dotted curve in Fig. 4) leads to a better agreement for low energies and confirms the similarity of the different curves with $V_{\text {init }}=V_{\text {meas. }}$. Hence no spatial variation of the total defect density is observed from these measurements.

\section{CONCLUSIONS}

Additional information about grading and metastable effects can be gained by performing admittance spectroscopy under different bias voltages. It is important to discriminate between effects due to the grading and due to metastabilities. This can be achieved by varying the applied voltage during the initial state (whilst creating the metastable state of the defect) and during the measurement independently or simultaneously.

Admittance spectroscopy measurements performed under different bias voltages on a flexible CIGS solar cell indicate the presence of a metastable defect level around $0.1-0.2 \mathrm{eV}$. This level could be assigned to a donor configuration state of an intrinsic DX center. The influence of band gap grading is visible in a shift of the activation energy with applied voltage, a spatial variation in the defect density could not be detected.

\section{ACKNOWLEDGEMENTS}

We acknowledge the support of the Research Foundation - Flanders (K.D., FWO Ph.D. fellowship) and of the IWT project 080407.

\section{REFERENCES}

[1] T. Walter, et al., "Determination of defect distributions from admittance measurements and application to $\mathrm{Cu}(\mathrm{In}, \mathrm{Ga}) \mathrm{Se}_{2}$ based heterojunctions," Journal of Applied Physics 80, pp. 4411-4420, Oct 1996.

[2] K. Decock, et al., "Defect distributions in thin film solar cells deduced from admittance measurements under different bias voltages," unpublished.

[3] K. Decock, et al., "Accuracy of defect distributions measured by bias dependent admittance spectroscopy on thin film solar cells," in IEEE PVSC Area 8, Seattle, 2011. 
[4] A. Chirilă, et al., "Cu(In,Ga)Se $\mathrm{Se}_{2}$ solar cell grown on flexible polymer substrate with efficiency exceeding 17\%," Progress in Photovoltaics: Research and Applications pp. n/a-n/a, 2011.

[5] P. Zabierowski, "Electrical characterization of $\mathrm{Cu}(\mathrm{In}, \mathrm{Ga}) \mathrm{Se}_{2}$-based thin film photovoltaic devices," in Thin Film Solar Cells: Current Status and Future Trends, A. Bosio and A. Romeo, Eds., ed: Nova Science Publishers, 2010.

[6] S. Lany and A. Zunger, "Light- and bias-induced metastabilities in $\mathrm{Cu}(\mathrm{In}, \mathrm{Ga}) \mathrm{Se}_{2}$ based solar cells caused by the $\left(\mathrm{V}_{\mathrm{Se}}-\mathrm{V}_{\mathrm{Cu}}\right)$ vacancy complex," Journal of Applied Physics 100, p. 113725, Dec 2006.

[7] S. Lany and A. Zunger, "Intrinsic DX centers in ternary chalcopyrite semiconductors," Physical Review Letters 100, p. 016401, Jan 2008.

[8] J. H. Scofield, "Effects of series resistance and inductance on solar cell admittance measurements," Solar Energy Materials and Solar Cells 37, pp. 217-233, May 1995. 